\title{
Longitudinal dispersion of orbital angular momentum modes in high-gain free-electron lasers
}

\author{
Erik Hemsing, ${ }^{1}$ Agostino Marinelli, ${ }^{2,3}$ Sven Reiche, ${ }^{1}$ and James Rosenzweig ${ }^{1}$ \\ ${ }^{1}$ Particle Beam Physics Laboratory, Department of Physics and Astronomy, University of California Los Angeles, \\ Los Angeles, California 90095, USA \\ ${ }^{2}$ Universita degli Studi di Roma "La Sapienza", Via Antonia Scarpa 14, Rome, 00161, Italy \\ ${ }^{3}$ INFN-LNF, via E. Fermi, 40-00044 Frascati, Rome, Italy
}

(Received 30 May 2008; published 30 July 2008)

\begin{abstract}
The physical effects of optical mode dispersion in the electron beam of a free-electron laser are investigated for modes that carry orbital angular momentum. The analysis is performed using a derived equivalence between two different formulations that describe the radiation fields in the linear regime.
\end{abstract}

DOI: 10.1103/PhysRevSTAB.11.070704

PACS numbers: 41.60.Cr, 42.50.Tx

\section{INTRODUCTION}

The coherent electromagnetic (EM) signal field generated in a free-electron laser (FEL) is optically guided within the source electron beam (e-beam) during exponential gain [1-3]. In cases where there is significant guiding of the radiation field, it can be useful to describe the FEL light as a sum over eigenmodes of a virtual waveguide structure [4,5]. This allows one to efficiently investigate the coupling and propagation characteristics of specific EM mode structures over many diffracting lengths. It also permits, through the type of virtual waveguide geometry chosen, flexibility in the form of the expansion mode basis so that particular modes of interest may be examined in detail or so that a specific basis set can be chosen to optimally suit a given FEL geometry. The eigenmodes of a quadratic index fiber, for example, are particularly useful as a virtual dielectric waveguide model because they are composed of composite Gaussian functions (typically Hermite-Gaussian or Laguerre-Gaussian functions) that also arise in the solutions to the paraxial wave equation for free-space propagation $[6,7]$. This correspondence establishes a useful connection between free-space modes and the optically guided modes of the FEL, providing a model that not only can describe optical mode propagation from startup through high gain, but also both the input (seeding) and output radiation characteristics.

In this framework, the coupling and propagation characteristics of well-known Laguerre-Gaussian (LG) modes can be studied explicitly for FELs. These modes are of particular interest since, for higher-order azimuthal modes, they are known to possess a well-defined value of orbital angular momentum (OAM) as a result of an azimuthal component of the linear photon momentum [8]. Modes of this type may be particularly relevant for study with next-generation $\mathrm{x}$-ray FELs which will have the ability to probe the structure of matter on short length and time scales. For future FEL light sources with, for example, flexibility in the polarization, the ability to generate intense higher-order LG modes would further extend the opera- tional capabilities. Advances in LG mode amplification and transport in FELs may also provide additional experimental versatility for potential applications like microscopy [9], information encoding [10], and entanglement schemes [11]. Coherent OAM modes also allow the possibility of light-driven micromechanical devices or the use of torque from photons as an exploratory tool [12].

The amplification of OAM modes in an FEL at the fundamental frequency has been investigated previously using a linear fluid model to describe the evolution of the ebeam in the cold beam limit [13]. This approach utilizes a specific case of the general formulation presented in [5] where the signal fields are described through a general expansion consisting of dielectric waveguide eigenmodes. The e-beam is ponderomotively coupled to the fields and a set of coupled 3D excitation and evolution equations are derived. The FEL eigenmode equations, or supermode solutions, are obtained by examining the fixed-profile solutions of the mode amplitudes, such that the individual mode coefficients in the field expansion are constant with respect to each other, and the overall field grows exponentially along the undulator. This description utilizes the advantages of the modal description technique wherein the characteristics of single modes can be studied in detail. Without the effects of energy spread, however, this approach is limited to cold e-beams. For optical wavelength FELs, analysis in the cold beam limit is often adequate. With the advent of shorter wavelength, high-gain systems, however, it is critical to include thermal effects in describing the evolution of the electron beam in the FEL.

In contrast to the fluid analysis, in the alternate formalism presented in Ref. [14], the general 3D solution for the field evolution is derived from a simultaneous solution of the Vlasov equation that describes the evolution of the e-beam distribution function for an arbitrary energy spread and Maxwell's equations in the paraxial limit. Eigensolutions for the FEL system are obtained by examining separable field solutions that have a fixed transverse profile and an exponentially growing amplitude. In this way, thermal effects in the longitudinal dimension are 
included in the description of the electron beam dynamics component of the FEL instability.

Both formulations $[5,14]$ describe the evolution both of the e-beam and the signal fields, beginning from the startup period and continuing through the linear growth regime. Both assume that the transverse charge density gradient in the field polarization direction is negligible $c^{2} \nabla_{i} \rho_{e} \ll$ $\partial_{t} J_{i}$, and that the transverse e-beam size, which is fixed along the undulator length (no active transverse rearrangement of the electrons), is large compared to the microbunching wavelength in the moving frame, $r_{0}>\gamma_{z} \lambda$.

In this work, in order to establish a solid connection between the two formalisms and to explore the interaction of multiple mode structures in the presence of an energy spread on the e-beam, the field evolution integral from [14] is reexpressed through an expansion of the field as a sum over dielectric waveguide eigenmodes. This provides: (a) a unified description that shows the equivalence between the formalisms in the linear gain regime, and (b) a compact set of mode coupled equations used to study the amplification and coupling to OAM modes in the FEL with the effects of energy spread included. The latter is a useful extension to results presented in Ref. [13], which concentrated only on a cold e-beam. The resulting equations are then used to explore the unique effects of longitudinal dispersion of OAM modes in the FEL, which arise due to the helical transverse phase dependence and mode-dependent coupling characteristics.

\section{INTEGRO-DIFFERENTIAL SOLUTION}

The integro-differential equation for the FEL field amplitudes $\tilde{E}_{s}$ derived in Ref. [14] is (in cgs units)

$$
\begin{aligned}
\nabla_{\perp}^{2} \tilde{E}_{s}+2 i \frac{\omega}{c} \frac{\partial \tilde{E}_{s}}{\partial z}= & i j_{0}\left(\mathbf{r}_{\perp}\right) \int_{0}^{z} d z^{\prime}\left\{\frac{2 \pi e}{c^{2}} \theta_{s}^{2} \omega \tilde{E}_{s}\left(\mathbf{r}_{\perp}, z^{\prime}\right)\right. \\
& \left.+\frac{4 \pi e}{\omega}\left[\nabla_{\perp}^{2} \tilde{E}_{s}+2 i \frac{\omega}{c} \frac{\partial \tilde{E}_{s}}{\partial z^{\prime}}\right]\right\} \\
& \times \int_{-\infty}^{\infty} d P \frac{d F}{d P} e^{i\left[-\theta+\left(\omega / \gamma_{2}^{2} \mathcal{E}_{0} c\right) P\right]\left(z^{\prime}-z\right)},
\end{aligned}
$$

where the transverse field of the amplified wave in the undulator is $E_{\perp}(\mathbf{r}, t)=\tilde{E}_{s}\left(\mathbf{r}_{\perp}, z\right) e^{i \omega(z / c-t)}, j_{0}\left(\mathbf{r}_{\perp}\right)$ is the ebeam current density, $\theta_{s}=K / \gamma=e|H| / \mathcal{E}_{0} k_{w}$ is the rotation angle in a helical undulator with peak magnetic field amplitude $|H|, k_{w}$ is the undulator lattice wave number, $\mathcal{E}_{0}=\gamma m c^{2}$ is the nominal energy of an electron, $\theta=$ $k / 2 \gamma_{z}^{2}-k_{w} \simeq \omega / v_{z_{0}}-k-k_{w}$ is the detuning parameter for a free-space paraxial wave, $\gamma_{z}^{-2}=\gamma^{-2}+\theta_{s}^{2}, v_{z_{0}}$ is the mean axial velocity of the e-beam, $k_{z}=k=\omega / c$ is the free-space wave number and $P=\mathcal{E}-\mathcal{E}_{0}$. The energy spread function $F(P)$ satisfies $\int F d P=1$.

Equation (1) describes the self-consistent excitation and evolution of the amplified radiation fields from the source e-beam during the FEL interaction, including the effects of longitudinal space charge and energy spread. The field amplitudes are slowly varying functions of $z$, and can be expressed as the product of two functions: $\tilde{E}_{s}\left(\mathbf{r}_{\perp}, z\right)=$ $\Phi\left(\mathbf{r}_{\perp}\right) \exp (\Lambda z)$ for solutions of the high-gain FEL supermode, which propagates self-similarly in the transverse dimension with an exponentially growing amplitude.

\section{A. Field expansion}

In the field expansion approach from [5], the transverse field is described by a sum of dielectric waveguide eigenmodes:

$$
\mathbf{E}_{\perp}(\mathbf{r}, t)=\sum_{q} C_{q}(z) \tilde{\mathcal{E}}_{\perp q}\left(\mathbf{r}_{\perp}\right) e^{i\left(k_{z q} z-\omega t\right)},
$$

where $k_{z q}$ is the axial wave number of the $q$ th mode and $C_{q}(z)$ is the slowly growing amplitude coefficient. The differences between the wave numbers of the modes leads to modal dispersion in the medium (and in the FEL), which also means that the detuning varies with the mode number. The transverse components of the modes are eigenfunctions of the dielectric equation:

$$
\nabla_{\perp}^{2} \tilde{\mathcal{E}}_{\perp q}\left(\mathbf{r}_{\perp}\right)+\left[n\left(\mathbf{r}_{\perp}\right)^{2} k^{2}-k_{z q}^{2}\right] \tilde{\mathcal{E}}_{\perp q}\left(\mathbf{r}_{\perp}\right)=0,
$$

and are determined by the form of the refractive index $n\left(\mathbf{r}_{\perp}\right)$. It is assumed that the fiber is weakly guiding and has a "slow" transverse variation $\left(\nabla n^{2} \ll k\right)$. The eigenmodes also form a complete and orthogonal basis set, with orthogonality defined as

$$
\left\langle\tilde{\mathcal{E}}_{\perp q} \mid \tilde{\mathcal{E}}_{\perp q^{\prime}}\right\rangle=\iint \tilde{\mathcal{E}}_{\perp q}^{*} \tilde{\mathcal{E}}_{\perp q^{\prime}} \mathbf{d}^{2} \mathbf{r}_{\perp}=\delta_{q, q^{\prime}}|A|^{2},
$$

where $A$ is a normalization constant that may carry units. The mode power is $\mathrm{P}_{q}(z)=k_{z q}|A|^{2}\left|C_{q}(z)\right|^{2} / 8 \pi k$.

The use of waveguide eigenmodes in the self-consistent equations requires a modified form for Eq. (1) because the mode-dependent wave number $k_{z q}$ alters the form of the paraxial approximation since $k_{z q} \neq k$. Using the dielectric waveguide eigenmodes in Eq. (2), the modified integrodifferential equation becomes

$$
\begin{aligned}
& \sum_{q} e^{i k_{z q} z}\left[\nabla_{\perp}^{2}+k^{2}-k_{z q}^{2}+2 i k_{z q} \frac{d}{d z}\right] C_{q}(z) \tilde{\mathcal{E}}_{\perp q}\left(\mathbf{r}_{\perp}\right) \\
& =i j_{0}\left(\mathbf{r}_{\perp}\right) \int_{0}^{z} d z^{\prime} \sum_{q} e^{i k_{z q} z^{\prime}}\left\{\frac{2 \pi e k}{c} \theta_{S}^{2} C_{q}\left(z^{\prime}\right) \tilde{\mathcal{E}}_{\perp q}\left(\mathbf{r}_{\perp}\right)\right. \\
& \left.\quad+\frac{4 \pi e}{\omega}\left[\nabla_{\perp}^{2}+k^{2}-k_{z q}^{2}+2 i k_{z q} \frac{d}{d z^{\prime}}\right] C_{q}\left(z^{\prime}\right) \tilde{\mathcal{E}}_{\perp q}\left(\mathbf{r}_{\perp}\right)\right\} \\
& \quad \times \int_{-\infty}^{\infty} d P \frac{d F}{d P} e^{i\left[-\theta-k+\left(k / \gamma_{z}^{2} \mathcal{E}_{0}\right) P\right]\left(z^{\prime}-z\right)} .
\end{aligned}
$$

The dielectric eigenmode relation in Eq. (3) is inserted to remove the transverse Laplacian term and simplify using orthogonality of the basis functions. The current density is written as $j_{0}\left(\mathbf{r}_{\perp}\right)=e c \mathrm{n}_{0} f\left(\mathbf{r}_{\perp}\right)$, where $\mathrm{n}_{0}$ is the electron 
density and $f\left(\mathbf{r}_{\perp}\right)$ is the transverse profile distribution function. The relativistic plasma wave number $\theta_{p}=$ $\sqrt{4 \pi \mathrm{n}_{0} e^{2} / \mathcal{E}_{0} \gamma_{z}^{2}}$ is identified and Eq. (5) becomes

$$
\begin{aligned}
\frac{d}{d z} C_{q}(z)= & \sum_{q^{\prime}}\left[-i C_{q^{\prime}}(z) \mathbb{K}_{q, q^{\prime}}^{d} e^{i\left(k_{z q^{\prime}}-k_{z q}\right) z}\right. \\
& +\frac{\theta_{p}^{2} \mathcal{E}_{0} \gamma_{z}^{2}}{k} \int_{0}^{z} d z^{\prime} e^{i\left(k_{z q^{\prime}} z^{\prime}-k_{z q} z\right)} \\
& \times\left\{\frac{k^{2} \theta_{s}^{2}}{4 k_{z q}} \mathbb{F}_{q, q^{\prime}}-\sum_{q^{\prime \prime}} \frac{k_{z q^{\prime \prime}}}{k_{z q}} \mathbb{F}_{q, q^{\prime \prime}} \mathbb{K}_{q^{\prime \prime}, q^{\prime}}\right. \\
& \left.+i \frac{k_{z q^{\prime}}}{k_{z q}} \mathbb{F}_{q, q^{\prime}} \frac{d}{d z^{\prime}}\right\} C_{q^{\prime}}\left(z^{\prime}\right) \\
& \left.\times \int_{-\infty}^{\infty} d P \frac{d F}{d P} e^{i\left[-\theta-k+\left(k / \gamma_{z}^{2} \mathcal{E}_{0}\right) P\right]\left(z^{\prime}-z\right)}\right]
\end{aligned}
$$

This equation describes the evolution of the waveguide mode amplitudes from the FEL startup through the highgain regime. It can be used to solve for the evolution of individual modes along the undulator for a given energy spread $F(P)$. The e-beam/mode overlap coefficient, or generalized "filling factor" is defined as

$$
\mathbb{F}_{q, q^{\prime}}=\frac{1}{|A|^{2}}\left\langle\tilde{\mathcal{E}}_{\perp q}\left|f\left(\mathbf{r}_{\perp}\right)\right| \tilde{\mathcal{E}}_{\perp q^{\prime}}\right\rangle,
$$

which can be computed for an arbitrary form of the e-beam profile function $f\left(\mathbf{r}_{\perp}\right)$. With an LG mode basis it is straightforward to compute $\mathbb{F}_{q, q^{\prime}}$ analytically for several different transverse e-beam profiles, including Gaussian, parabolic, and flattop distributions. The coupling between modes in the virtual dielectric for a specified form of the refractive index in Eq. (3) is given by

$$
\mathbb{K}_{q, q^{\prime}}=\frac{k^{2}}{2 k_{z q}|A|^{2}}\left\langle\tilde{\mathcal{E}}_{\perp q}\left|n\left(\mathbf{r}_{\perp}\right)^{2}-1\right| \tilde{\mathcal{E}}_{\perp q^{\prime}}\right\rangle .
$$

The sum over the product of the elements of $\mathbb{F}_{q, q^{\prime \prime}}$ and $\mathbb{K}_{q^{\prime \prime}, q}$ in the second term in the $z$-integrand results from the integral

$$
\begin{aligned}
& \frac{k^{2}}{2 k_{z q}|A|^{2}}\left\langle\tilde{\mathcal{E}}_{\perp q}\left|f\left(\mathbf{r}_{\perp}\right)\left[n\left(\mathbf{r}_{\perp}\right)^{2}-1\right]\right| \tilde{\mathcal{E}}_{\perp q^{\prime}}\right\rangle \\
& =\sum_{q^{\prime \prime}} \frac{k^{2}}{2 k_{z q}|A|^{4}}\left\langle\tilde{\mathcal{E}}_{\perp q}\left|f\left(\mathbf{r}_{\perp}\right)\right| \tilde{\mathcal{E}}_{\perp q^{\prime \prime}}\right\rangle\left\langle\tilde{\mathcal{E}}_{\perp q^{\prime \prime}}\left|n\left(\mathbf{r}_{\perp}\right)^{2}-1\right| \tilde{\mathcal{E}}_{\perp q^{\prime}}\right\rangle \\
& =\sum_{q^{\prime \prime}} \frac{k_{z q^{\prime \prime}}}{k_{z q}} \mathbb{F}_{q, q^{\prime \prime}} \mathbb{K}_{q^{\prime \prime}, q^{\prime}} .
\end{aligned}
$$

In the second step the identity operator $\sum_{q^{\prime \prime}}\left|\tilde{\mathcal{E}}_{\perp q^{\prime \prime}}\right\rangle\left\langle\tilde{\mathcal{E}}_{\perp q^{\prime \prime}}\right|$ has been inserted with the condition that the eigenmode basis forms a complete set.
Solutions to Eq. (6) for a sufficiently long interaction length are the supermode solutions of the FEL. The supermode field profile maintains a fixed transverse distribution as the resonant interaction between the active source ebeam and the signal field results in optical guiding of the radiation field, balancing the diffraction effects. The guided supermode (SM) fields are identified by a fixed superposition of the expansion modes that grows in amplitude exponentially along $z$ :

$$
\tilde{\mathbf{E}}_{\mathrm{SM}}(\mathbf{r})=\left[\sum_{q} b_{q} \tilde{\mathcal{E}}_{\perp q}\left(r_{\perp}\right)\right] e^{i k_{\mathrm{SM}} z}
$$

This expression is obtained by a simple transformation of the mode amplitude coefficients,

$$
C_{q}(z)=b_{q} e^{i\left(k_{\mathrm{SM}}-k_{z q}\right) z}
$$

where the complex supermode wave number is defined as a modification of the free-space wave number by a perturbation associated with the FEL interaction,

$$
k_{\mathrm{SM}}=k+\tilde{\delta k}
$$

Equation (11) can be inserted into Eq. (6), transforming it into an algebraic relationship for a direct calculation of the supermode coefficients $b_{q}$. This transformation allows a straightforward evaluation of the integral over the longitudinal coordinate, since all the dependence on $z$ is compactly located in the exponential term. For each $b_{q}$,

$$
\begin{aligned}
\left(\tilde{\delta k}-\Delta k_{q}\right) b_{q}= & -\sum_{q^{\prime}} b_{q^{\prime}}\left[\mathbb{K}_{q, q^{\prime}}^{d}+S(\tilde{\delta k}, \theta) \frac{\theta_{p}^{2} \mathcal{E}_{0} \gamma_{z}^{2}}{k}\right. \\
& \times\left\{\frac{k^{2} \theta_{s}^{2}}{4 k_{z q}} \mathbb{F}_{q, q^{\prime}}-\sum_{q^{\prime \prime}} \frac{k_{z q^{\prime \prime}}}{k_{z q}} \mathbb{F}_{q, q^{\prime \prime}} \mathbb{K}_{q^{\prime \prime}, q^{\prime}}\right. \\
& \left.\left.-\frac{k_{z q^{\prime}}}{k_{z q}}\left(\tilde{\delta k}-\Delta k_{q}\right) \mathbb{F}_{q, q^{\prime}}\right\}\right],
\end{aligned}
$$

where $\Delta k_{q}=k_{z q}-k$. The effects of the energy spread and detuning are expressed as a single function $S(\tilde{\delta k}, \theta)$, defined by the integral

$$
S(\tilde{\delta k}, \theta)=\int_{-\infty}^{\infty} d P \frac{d F / d P}{\tilde{\delta k}-\theta+\frac{k}{\gamma_{z}^{2} \mathcal{E}_{0}} P} .
$$

The e-beam mode coupling parameter for dominantly transverse modes is closely approximated in the limit $k \simeq$ $k_{z q^{\prime}} \gg k_{w}$ by $[4,5]$

$$
\mathbb{Q}_{q, q^{\prime}}=\theta_{p}^{2} \frac{k^{2} \theta_{s}^{2}}{4 k_{z q}} \mathbb{F}_{q, q^{\prime}} .
$$

This characterizes the coupling between the e-beam and the waveguide modes. For a planar undulator geometry, $\theta_{s}^{2}=K^{2} / 2 \gamma^{2}$, and $\mathbb{Q}_{q, q^{\prime}}$ must be multiplied by the factor $\mathrm{JJ}=\left[J_{0}(\alpha)-J_{1}(\alpha)\right]^{2}$, where $J_{0}$ and $J_{1}$ are Bessel functions of the first kind and $\alpha=K^{2} /\left(4+2 K^{2}\right)$. 
It is useful to define the parameter $\mathbb{M}_{q, q^{\prime}}=$ $\left(k_{z q^{\prime}} / k_{z q}\right) \mathbb{F}_{q, q^{\prime}}$ and to write Eq. (13) in matrix form. The matrix elements are indexed by the mode subscripts: the matrix $\underline{\mathbb{Q}}$ contains elements $\mathbb{Q}_{q, q^{\prime}}$, the matrix $\underline{\underline{\Delta k}}$ has elements $\Delta k_{q} \delta_{q, q^{\prime}}$, and so on. The matrix $\underline{\underline{\mathbf{I}}}$ is the identity. Equation (13) is then

$$
\left[\left[\underline{\underline{\mathbf{I}}} \frac{k}{S(\tilde{\delta k}, \theta) \mathcal{E}_{0} \gamma_{z}^{2}}-\theta_{p}^{2} \underline{\underline{\mathbb{M}}}\right][\underline{\underline{\mathbf{I}}} \tilde{\delta k}+\underline{\underline{\mathbb{K}}}-\underline{\underline{\Delta k}}]+\underline{\underline{\mathbb{Q}}}\right] \underline{b}=\underline{0} .
$$

The supermode coefficients $b_{q}$, which are elements of the column vector $\underline{b}$ (or matrix $\underline{b}$ if the index is double valued: $q=\{p, l\}$ ), are given by solutions to Eq. (16). The dominant, or highest gain, supermode coefficients correspond to the solution that yields the most negative value of $\operatorname{Im}\{\tilde{\delta k}\}$, defined to be $\tilde{\delta k_{i}}$. The full complex valued solution $\tilde{\delta k}=$ $\tilde{\delta k_{r}}+i \tilde{\delta k_{i}}$ is inserted, with the corresponding set of eigenvalue coefficients $b_{q}$, into Eq. (10) to yield the dominant supermode field of the FEL. The 3D power gain length, or e-folding length, is given by $L_{G}=1 /\left|2 \tilde{\delta} k_{i}\right|$.

For a monochromatic beam the energy distribution function is given by $F(P)=\delta\left(P-P_{1}\right)$. The energy spread function in Eq. (14) is then

$$
S(\tilde{\delta k}, \theta)=\frac{k}{\mathcal{E}_{0} \gamma_{z}^{2}}\left(\tilde{\delta k}-\theta+\frac{k}{\mathcal{E}_{0} \gamma_{z}^{2}} P_{1}\right)^{-2},
$$

with $P_{1}=0$ the cold beam energy spread function from Eq. (17) is inserted into the supermode matrix equation (16). In determinant form it becomes

$$
\left|\left[\underline{\underline{\mathbf{I}}}(\tilde{\delta k}-\theta)^{2}-\theta_{p}^{2} \underline{\underline{\mathbb{M}}}\right][\underline{\underline{\mathbf{I}}} \tilde{\delta k}+\underline{\underline{\mathbb{K}}}-\underline{\underline{\Delta k}}]+\underline{\underline{\mathbb{Q}}}\right|=0 .
$$

This is precisely the supermode equation derived using the linear e-beam fluid model in Ref. [5]. The effects of longitudinal space charge are included through the $\theta_{p}^{2} \underline{\underline{M}}$ term. In the 1D limit the matrix elements are degenerate: $\underline{\underline{\mathbb{F}}} \rightarrow \underline{\underline{\mathbf{I}}}$ and $\underline{\underline{K}}, \underline{\underline{\Delta k}} \rightarrow \underline{\underline{\mathbf{0}}}$, and Eq. (18) effectively reduces to the familiar FEL cubic equation with gain parameter $\underline{\underline{\mathbb{Q}}} \rightarrow$ $\underline{\underline{\mathbf{I}}} \mathbb{Q}=\underline{\underline{\mathbf{I}}}\left(\theta_{p} \theta_{s}\right)^{2} k / 4=\underline{\underline{\mathbf{I}}}\left(2 k_{w} \rho\right)^{3}$, where $\rho$ is the wellknown Pierce parameter often used in FEL theory [15].

The general supermode matrix equation in (16) can be written in eigenvalue form as

$$
\underline{\underline{\mathbf{W}}} \underline{b}=\tilde{\lambda} \underline{b},
$$

where $\quad \underline{\underline{\mathbf{W}}}=\left[\underline{\underline{\mathbf{I}}} k / S \mathcal{E}_{0} \gamma_{z}^{2}-\theta_{p}^{2} \underline{\underline{\mathbb{M}}}\right][\underline{\underline{\mathbb{K}}}-\underline{\underline{\Delta k}}]-\tilde{\delta k} \theta_{p}^{2} \underline{\underline{\mathbb{M}}}+$ $\underline{\mathbb{Q}}$ with eigenvalue $\tilde{\lambda}=-k \tilde{\delta k} / \bar{S} \mathcal{E}_{0} \bar{\gamma}_{z}^{2}$ for a given $\overline{\tilde{\delta k}}$. If the waveguide expansion basis given by Eq. (3) is also the eigenmode basis of the FEL, then $\underline{\underline{\mathbf{W}}}$ is diagonal. Otherwise, the eigenvectors can be transformed by $\underline{b}=$ $\underline{\underline{\mathbf{T}}} \underline{u}$, where $\underline{\underline{\mathbf{T}}}^{-1} \underline{\underline{\mathbf{W}}} \underline{\underline{\mathbf{T}}}$ is a diagonal matrix and the vectors $\underline{u}$ are eigenvectors of the FEL system.

\section{MODE COUPLING AND DISPERSION}

Equation (6) describes the evolution of the individual waveguide mode amplitudes from the initial startup regime through the high-gain linear regime. Equations (16) and (19) can both be used to directly solve for the fixed-profile eigenmodes of the FEL. The coupling to guided Gaussian modes can be investigated by choosing an expansion composed of Laguerre-Gaussian (LG) (or Hermite-Gaussian) eigenmodes of a quadratic index medium $[7,16,17]$. These modes form a complete basis and can be used to investigate the amplification of transverse mode structures at the fundamental frequency like, for example, the hollow intensity profiles of azimuthal LG modes that carry orbital angular momentum. An axisymmetric e-beam profile that lacks a dominant OAM seed (either in input field or in the ebeam), exhibits equal coupling between the $l<0$ and $l>$ 0 OAM modes and the net amplified OAM in the optical beam is zero [see Eq. (15)]. Figure 1 shows such a scenario for model parameters of the SPARC FEL [18], depicting the dependence of the inverse gain length for $l=0,1$, and 2 modes on the energy spread for a Gaussian e-beam. These curves are obtained by finding the dominant solution to Eq. (16) with only a single azimuthal mode in the field expansion. This is possible for an axisymmetric beam since the OAM modes do not couple to each other. The curves in Fig. 1 therefore show the gain of the eigensolutions to Eq. (6) in the case of a single OAM mode defined as an initial value $\left[C_{p, l}(0)=1\right.$, for example, with $C_{p, l}(0)=0$ for $p \neq 0$ and $l \neq 1$ ]. The energy spread $F(P)$ is taken to be Lorentzian [being a reasonable approximation to a physical beam and giving an analytic solution to Eq. (14)], with a FWHM of $\delta \mathcal{E}$. It is clear from the plot that the modes with $l=0$ identify the dominant modes of the system at zero detuning, since they have the shortest gain lengths and thus the highest gain. This is attributed, in part, to the geometry of the field structure for $|l|>0$ modes

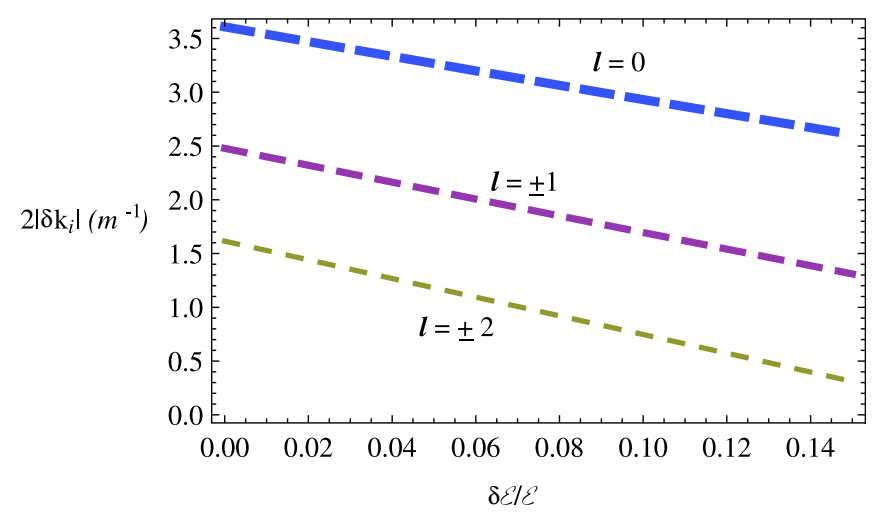

FIG. 1. (Color) Differential power for individual OAM modes on a Gaussian e-beam profile, with 11 radial modes included in the expansion. FEL model parameters are: $\theta_{p}=0.7 \mathrm{~m}^{-1}, \theta=$ $0, \gamma=300, K=1.8, I_{p}=500$ Amps peak current, $\lambda=$ $425 \mathrm{~nm}$. 
since they have zero field on-axis and shorter Rayleigh lengths for the same spot size than the fundamental Gaussian. Both effects conspire to reduce the effective coupling to the e-beam. It is possible, for certain FEL parameters, to detune such that the hollow OAM modes have a shorter gain length than the on-axis modes [14]. Further details regarding OAM mode amplification have also been explored elsewhere [13].

In addition to the increased gain length of OAM modes (and high-order modes in general) near resonance, the reduced coupling also results in reduced values for the effective optical guiding. This is given by the associated real part of the dominant solution to Eq. (18) as $\operatorname{Re}\{\tilde{\delta k}\}=$ $\tilde{\delta k_{r}}$. This gives an OAM mode-specific value for the effective guiding index of refraction defined as

$$
n_{\mathrm{eff}}=1+\frac{\tilde{\delta k_{r}}}{k} .
$$

Since the modes each have a different guiding index, they propagate with different phase velocities, leading to modal dispersion in the e-beam (Fig. 2). For modes with a significant transverse variation in phase, like OAM modes which have helical phases, this can be an experimentally observable effect as two modes shift in phase with respect to each other, causing portions of the transverse profile to change in intensity along the undulator axis. Figure 3 shows this effect for an OAM mode that is seeded and amplified simultaneously with the fundamental mode which grows due to a small, but nonzero value of prebunching. Such is a simplified case of seeding on a beam in the presence of SASE, when statistical noise on the beam at the resonant wavelength grows exponentially. The OAM mode in Fig. 3 is injected with enough power such that both modes have the same power at the undulator exit.

Evident from the plots in Fig. 3 are both the off-axis growth of the intensity which pushes the axial field null aside, and the rotation of the peak intensity spot along $z$ as both modes are amplified. Both effects are explained by the helical structure of phase of the OAM field. In general, the phase is such that amplification of the pure OAM mode tends to bunch the e-beam into a continuous helix (or multiply twisted helices for $|l|>1$ ) longitudinally. The fundamental mode also tends to bunch the beam, but into separated microbunches. With amplification of both an OAM mode with index $l$ and the fundamental mode in the system, the electrons are resonant on one side of the ebeam, but are $l \pi$ out of phase on the opposite side (Fig. 4). This means that there is higher growth of the signal field in the transverse position(s) where the electrons are resonant, and the intensity then grows off axis. This also explains why the evolution toward the fundamental does not begin with the peak intensity growing directly from the axial center of the beam, as would be the case if the initially dominant OAM mode were not present. The off-axis intensity bulge grows along the undulator both transversely and in amplitude due to the higher gain of the dominant mode, rotating about the axis as the two modes shift out of phase by $\psi=\left|\Delta n_{\text {eff }}\right| k \Delta z$, where $\Delta n_{\text {eff }}$ is the difference in the effective refractive index between the modes in Eq. (20), and $\Delta z$ is the length traveled along the undulator during high gain. Thus, OAM modes with azimuthal mode numbers differing by $\Delta l$ and with comparable amplitudes interfere constructively off axis to generate an intensity peak which rotates about the axis by $\psi / \Delta l$ radians over the length $\Delta z$. In addition, since the different pure modes also have different intrinsic radial profiles, the azimuthal motion of the intensity peak is accompanied by motion in the radial direction as the initially off-axis peak moves toward the radial maximum of the dominant mode of the systemtypically the axial center for an axisymmetric beam.

We note that the deviation from the simple microbunching profile of well-defined $l$-mode components is a measurable phenomenon. The signature of microbunching revealed through coherent transition radiation (CTR) has been well developed theoretically [19] and experimentally $[20,21]$ for Gaussian mode profiles. As such, CTR measurement is now a standard approach to diagnosis of beam microstructure. This established methodology may be straightforwardly extended to include microstructures induced wholly or in part by OAM modes. The theoretical basis of CTR measurement of helical beam microstructures is the subject of a future study.

For the cold beam scenario in Fig. 3, the calculated phase shift between modes is $\psi \simeq 2 \pi / 3$. This is calculated using the values of the refractive index for the OAM seed and the fundamental given by the $l=1$ and $l=0$ curves in Fig. 2. This phase shift is confirmed by visual inspection from the motion of the peak intensity spot which travels approximately $1 / 3$ of the way around the axis over an axial propagation distance of $\Delta z=3 L / 4$. An energy spread changes the amount of observed rotation. This is because

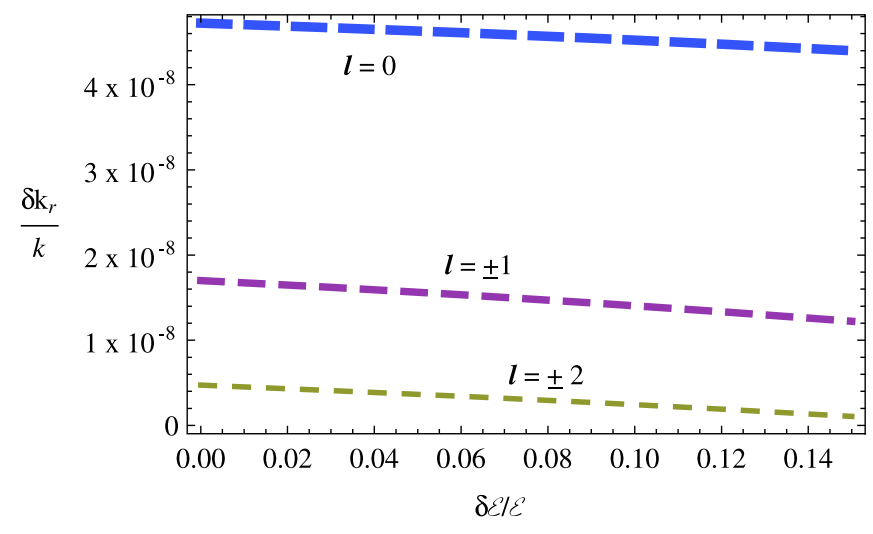

FIG. 2. (Color) Effective guiding due to the linear FEL interaction for OAM modes at resonance. Higher-order modes have a reduced coupling and are guided less strongly, and thus have a larger axial phase velocity through the e-beam. FEL model parameters are identical to Fig. 1. 
the phase difference between the modes is a function of $\Delta n_{\mathrm{eff}}$, which varies for different values of $\delta \mathcal{E}$. It is found here that $\left|\Delta n_{\text {eff }}\right|$ is larger (faster rotation) with increasing $\delta \mathcal{E}$ if the fundamental mode $l=0$ and an OAM mode $|l|>$ 0 are present.
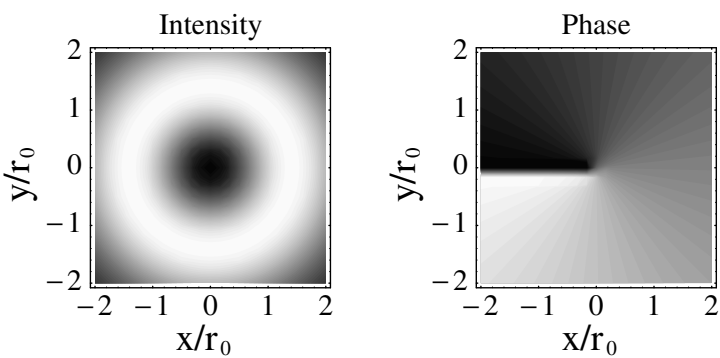

$\mathrm{Z}=0$
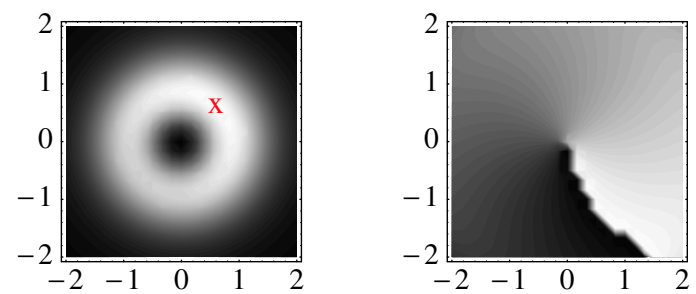

$\mathrm{z}=\mathrm{L} / 4$
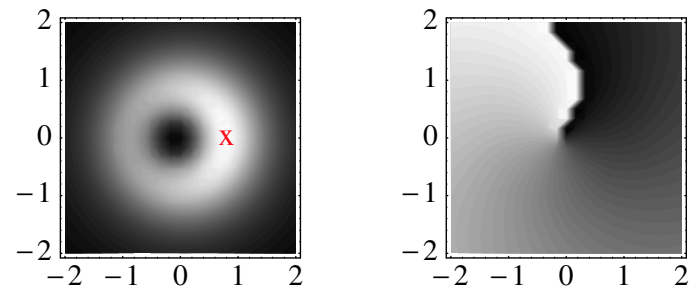

$\mathrm{z}=\mathrm{L} / 2$
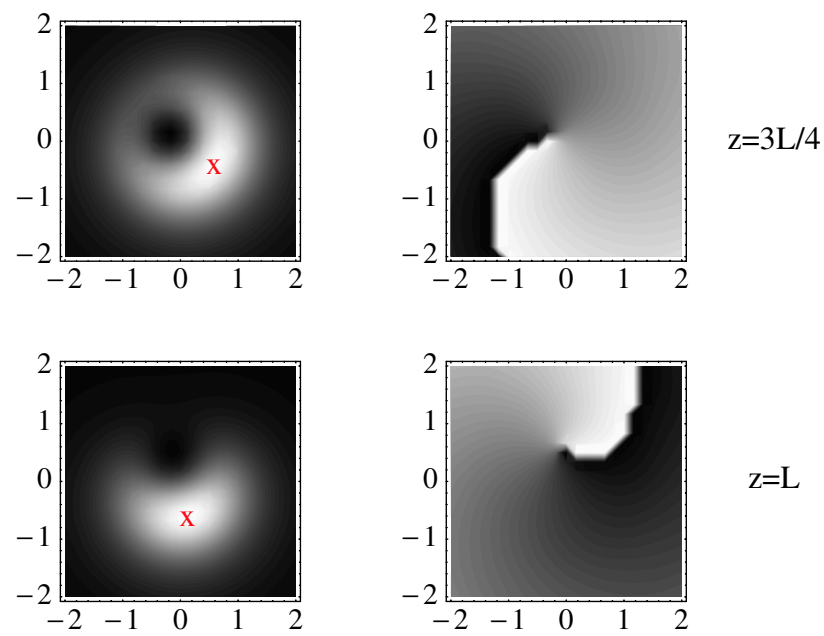

$\mathrm{Z}=\mathrm{L}$

FIG. 3. (Color) Intensity and phase along the undulator for an $l=1$ seed on a cold beam, prebunched at the fundamental. The fundamental mode grows faster than the OAM mode, appearing as a bright spot ( red $\mathrm{x}$ ) on the annular intensity pattern and eventually pushing the phase singularity, located at the field null, off to the side. The different effective phase velocities of the modes is manifest in the rotation of the bright spot along the undulator.
In Fig. 5 , an $l=2$ OAM mode is seeded onto the same prebunched beam as for Fig. 3, so $\Delta l=2$ between the amplified modes. The initial phase at $z=0$ is $\pi$ rotationally symmetric, so the e-beam that is bunched into a double helix is resonant with the fundamental at two off-axis locations which drives two intensity peaks appearing in opposition at $z=L / 4$. The peaks rotate along the undulator axis according to the $\psi \simeq 2 \pi \times(0.31)$ phase slippage between modes over $\Delta z=L / 2$ meters. This results in a rotation of $\psi / \Delta l \simeq 0.98$ radians $\left(\sim 56^{\circ}\right)$ before the peaks appear to merge. The direction of rotation is also in the same clockwise sense as in the seeding shown in Fig. 3, since both OAM seeds have positive values of $l$. The gain of the $l=2$ mode is less than the $l=1$ mode so the fundamental clearly dominates at $z=L$. This contrasts Fig. 3, where the influence of the OAM mode is still recognizable at the end. It is interesting to note that the field null for the $l=2$ mode is still essentially pushed aside by the dominant fundamental, but that the single phase singularity at the undulator entrance is broken into two vortices, each with topological charge 1 . This occurs in the interaction between the fundamental and all modes with $|l|>1$ due to the symmetry in phase about the axis; the fundamental peak evolves toward the center, fragmenting the phase singularities and taking their place on axis. Future investigations could shed light on this issue in detail, showing exactly how much of the total orbital angular momentum may be transferred to the e-beam in this process, and how much may become coupled to the spin off axis.

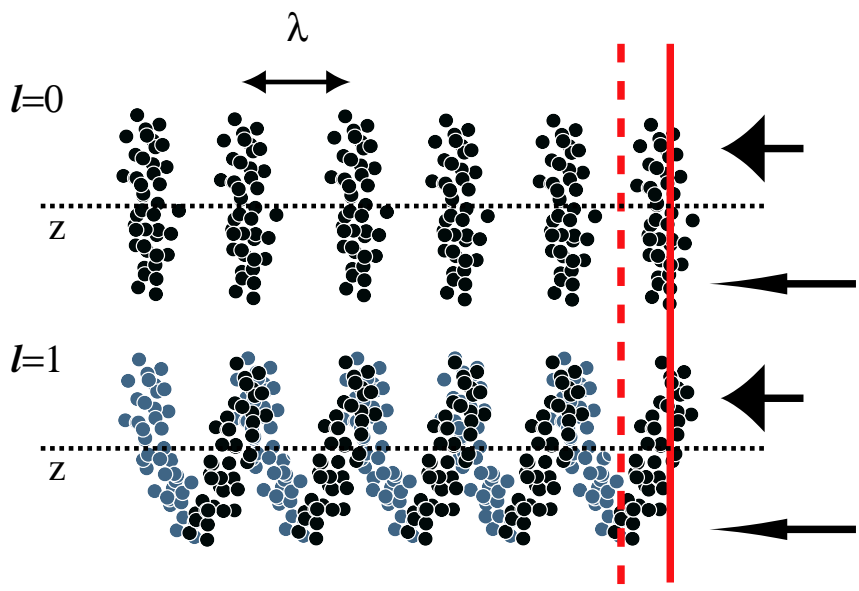

FIG. 4. (Color) Side by side schematic comparison of geometric bunching due to different modes. The fundamental mode (top) tends to generate separate bunches along the $z$ axis. The OAM modes (bottom) tend to bunch the beam in helices due to the azimuthal component of the ponderomotive phase. If both modes are simultaneously present, the electrons on one side of the ebeam (thick arrows) are resonant (solid vertical line), while those in a different transverse position (slender arrows) are bunched out of phase (dashed vertical line). The intensity therefore grows off axis, where the electrons are in phase. 

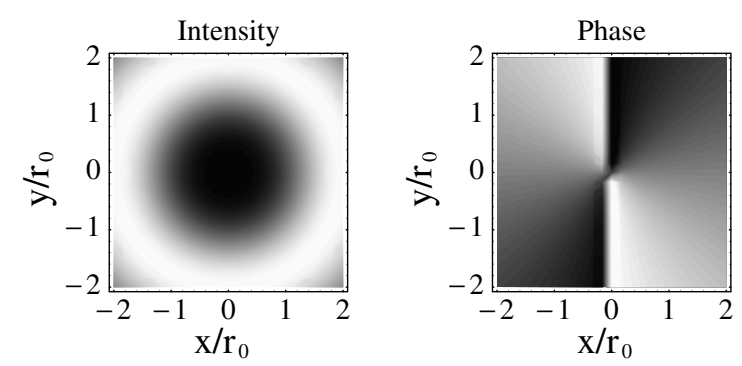

$\mathrm{Z}=0$
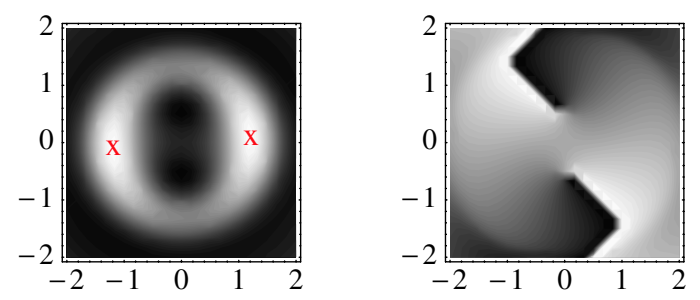

$\mathrm{Z}=\mathrm{L} / 4$
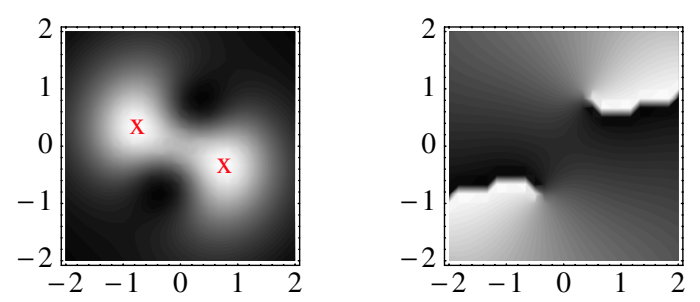

$\mathrm{z}=\mathrm{L} / 2$
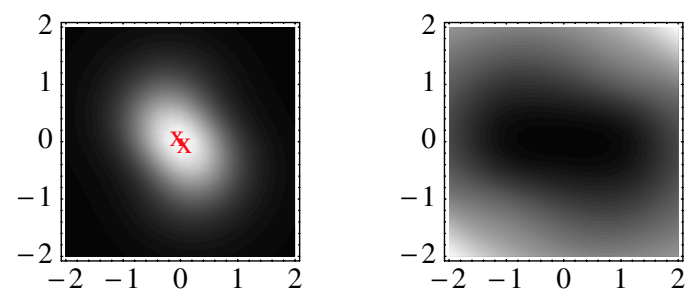

$\mathrm{z}=3 \mathrm{~L} / 4$
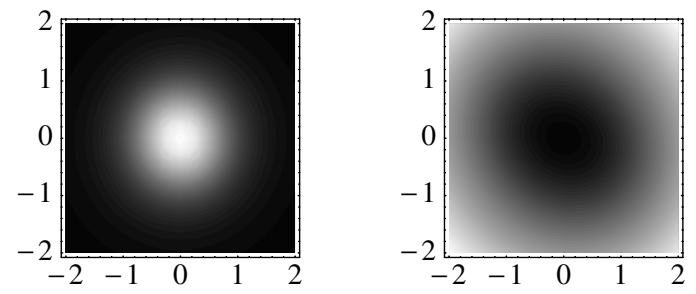

$\mathrm{Z}=\mathrm{L}$

FIG. 5. (Color) Intensity and phase along the undulator for an $l=2$ seed on a cold beam. The axial phase singularity is broken into two $l=1$ vortices as the fundamental grows.

In summary, an equivalence between two different descriptions of an FEL in the linear regime has been demonstrated, and used to explore the characteristics gain and guiding of OAM modes. The 3D integro-differential field equations obtained in Ref. [14] have been examined in the case that the signal field is written as a sum over eigenmodes of a dielectric waveguide. Self-similar guided fields are obtained in the linear gain regime from solutions to a determinant equation for fixed expansion mode coefficients. In the cold beam limit, these solutions reproduce the supermode equations derived in Ref. [5]. The correspondence between formalisms extends the versatility of the waveguide mode evolution and supermode equations derived using the linear fluid model approach in [5] to include the effects of energy spread. This enables an enhanced description of coupling to specific EM mode structures and realistic e-beams. An experimental scenario is suggested for the investigation of multiple pure mode structures during amplification which would clearly demonstrate the often hidden physical effects of mode dispersion, and hence unambiguously expose the effects of guiding due to the real part of the solution to the modified FEL cubic equation. Radiation in the form of CTR may also be used, in addition to the FEL emission, as a diagnostic to characterize the helical microbunching of the ebeam. Future theoretical work will be needed to extend the present analysis to include the effects of significant betatron motion and emittance effects, as well as the signature of helical bunching on CTR radiation.

\section{ACKNOWLEDGMENTS}

The authors would like to thank Professor Avi Gover for helpful discussions. This research was supported by grants from Department of Energy Basic Energy Science Contract No. DOE DE-FG02-07ER46272 and Office of Naval Research Contract No. ONR N00014-06-1-0925.

[1] N. M. Kroll, P. L. Morton, and M. N. Rosenbluth, IEEE J. Quantum Electron. 17, 1436 (1981).

[2] G. T. Moore, Nucl. Instrum. Methods Phys. Res., Sect. A 239, 19 (1985).

[3] E. T. Scharlemann, A. M. Sessler, and J. S. Wurtele, Phys. Rev. Lett. 54, 1925 (1985).

[4] Y. Pinhasi and A. Gover, Phys. Rev. E 51, 2472 (1995).

[5] E. Hemsing, A. Gover, and J. Rosenzweig, Phys. Rev. A 77, 063830 (2008).

[6] A. Siegman, Lasers (University Science Books, Sausalito, California, 1986).

[7] A. Yariv, Quantum Electronics (Wiley, New York, 1989), 3rd ed.

[8] L. Allen, M. W. Beijersbergen, R. J. C. Spreeuw, and J. P. Woerdman, Phys. Rev. A 45, 8185 (1992).

[9] P. Török and P. Munro, Opt. Express 12, 3605 (2004).

[10] G. Gibson, J. Courtial, M. Padgett, M. Vasnetsov, V. Pas'ko, S. Barnett, and S. Franke-Arnold, Opt. Express 12, 5448 (2004).

[11] E. Yao, S. Franke-Arnold, J. Courtial, M. J. Padgett, and S. M. Barnett, Opt. Express 14, 13089 (2006).

[12] L. Allen, S. M. Barnett, and M. J. Padgett, Optical Angular Momentum (Institute of Physics, Bristol, United Kingdom, 2003), ISBN 0750309016.

[13] E. Hemsing, A. Gover, and J. Rosenzweig, Phys. Rev. A 77, 063831 (2008).

[14] E. L. Saldin, E. A. Schneidmiller, and M. V. Yurkov, Opt. Commun. 97, 272 (1993). 
[15] R. Bonifacio, C. Pellegrini, and L. Narducci, Opt. Commun. 50, 373 (1984).

[16] O. Georg, Appl. Opt. 21, 141 (1982).

[17] L. Yu, W. Huang, M. Huang, Z. Zhu, X. Zeng, and W. Ji, J. Phys. A 31, 9353 (1998).

[18] D. Alesini et al., Nucl. Instrum. Methods Phys. Res., Sect. A 528, 586 (2004).
[19] J. Rosenzweig, G. Travish, and A. Tremaine, Nucl. Instrum. Methods Phys. Res., Sect. A 365, 255 (1995).

[20] A. Tremaine, J. B. Rosenzweig, S. Anderson, P. Frigola, M. Hogan, A. Murokh, C. Pellegrini, D. C. Nguyen, and R. L. Sheffield, Phys. Rev. Lett. 81, 5816 (1998).

[21] A. Tremaine et al., Phys. Rev. Lett. 88, 204801 (2002). 